\title{
Confinement and Processing Can Alter the Morphology and Periodicity of Bottlebrush Block Copolymers in Thin Films
}

Daniel F. Sunday, ${ }^{* 1}$ Moshe Dolejsi, ${ }^{2}$ Alice B. Chang, ${ }^{3}$ Lee J. Richter, ${ }^{1}$ Ruipeng Li,${ }^{4}$ R. Joseph Kline, ${ }^{1}$ Paul F. Nealey, ${ }^{2}$ Robert H. Grubbs ${ }^{3}$

1 Materials Science and Engineering Division, National Institute of Standards and Technology, 100 Bureau Drive, Gaithersburg, MD 20899

2 Institute for Molecular Engineering, University of Chicago, 5801 S Ellis Ave, Chicago, IL 60637

3 Division of Chemistry and Chemical Engineering, California Institute of Technology, Pasadena, California 91125, United States

4 National Synchrotron Light Source II, Brookhaven National Laboratory, Upton, NY 11973, United States

*Daniel.Sunday@nist.gov

\section{Supporting Information}

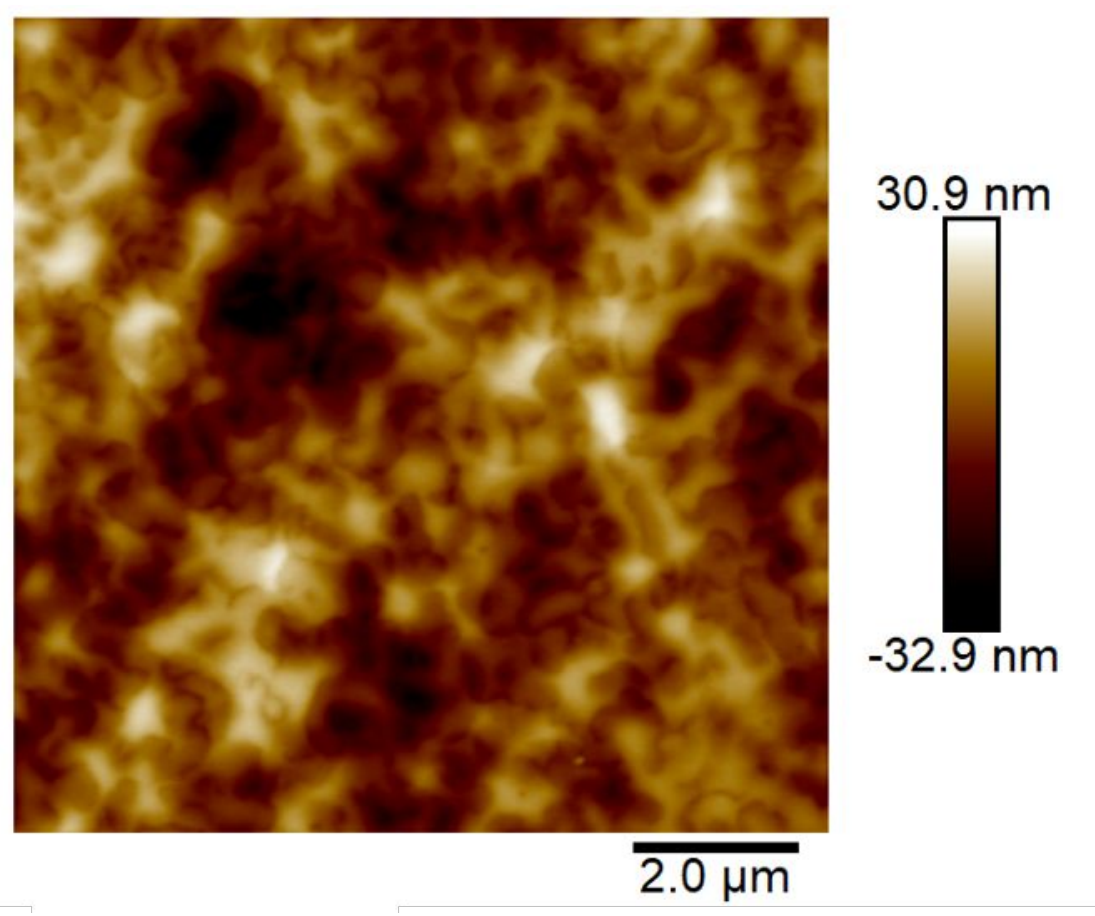

Figure S1: AFM height image of gPS- $b$-gPLA blade coated out of a THF solution at a thickness of $90 \mathrm{~nm}$ prior to annealing. 


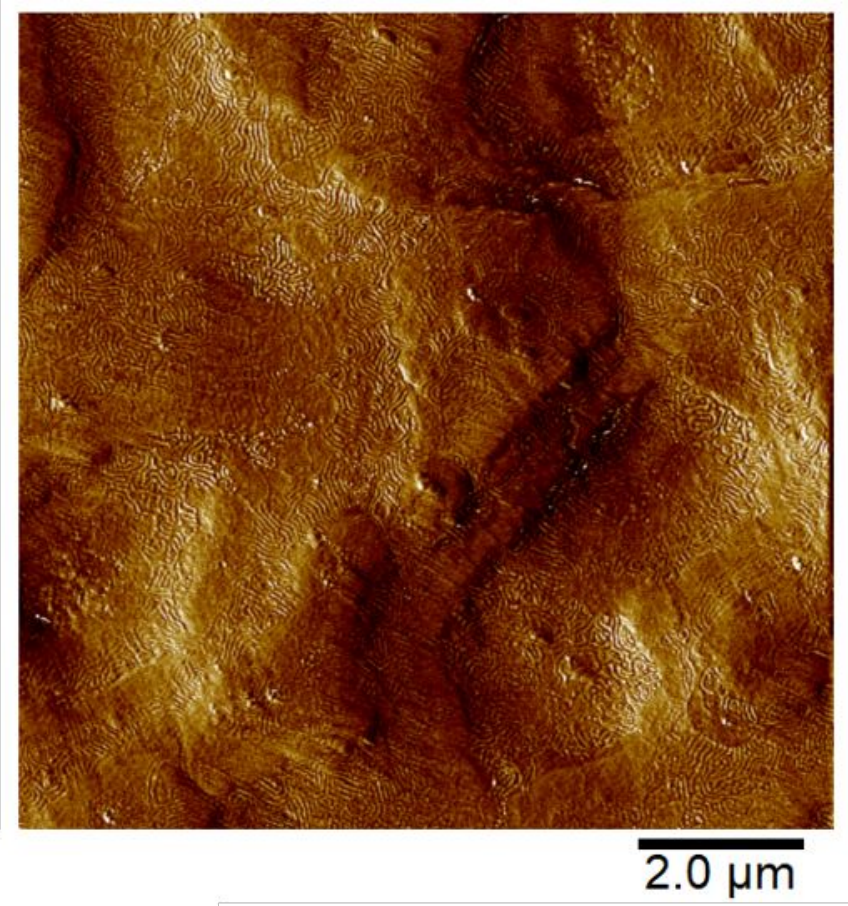

Figure S2: AFM phase image of gPS- $b$-gPLA blade coated out of a THF solution at a thickness of $110 \mathrm{~nm}$ after annealing at $165^{\circ} \mathrm{C}$ for $24 \mathrm{hrs}$.

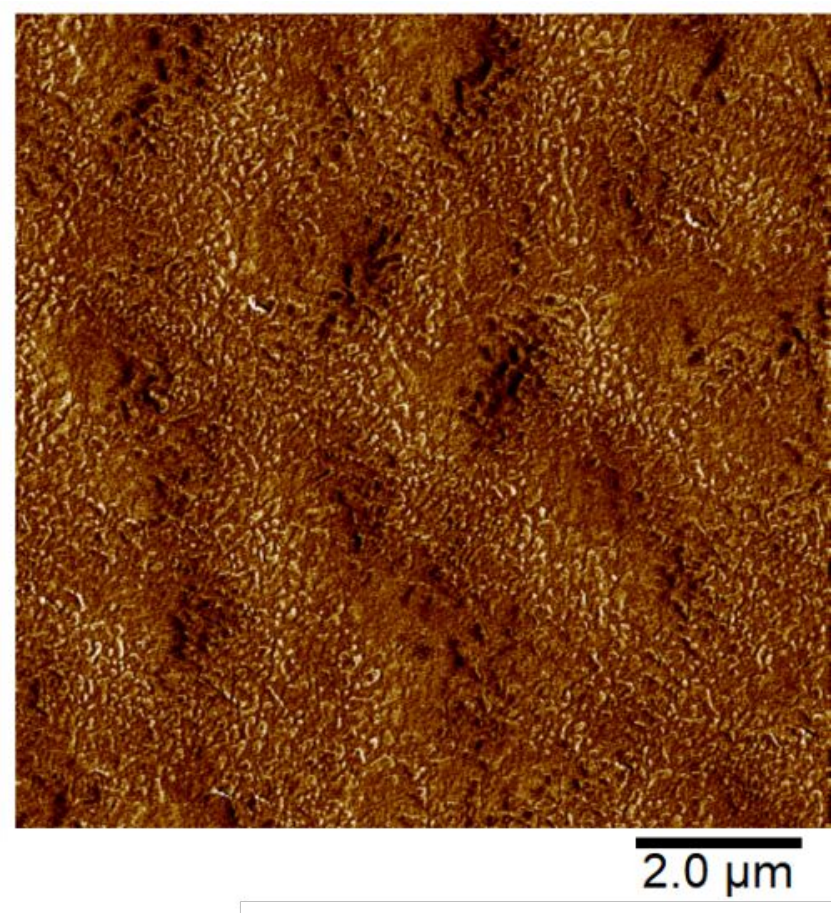

Figure S3: AFM phase image of gPS- $b$-gPLA blade coated out of a THF solution at a thickness of $50 \mathrm{~nm}$ after annealing at $165^{\circ} \mathrm{C}$ for $24 \mathrm{hrs}$. The PSD yields a broad peak centered around $85 \mathrm{~nm}$. 